\title{
Nanobodies and Antibodies for Duplexed EGFR/HER2 \\ Immunoassays Using Terbium-to-Quantum Dot FRET
}

Xue Qiu, ${ }_{1}$ K. David Wegner, ${ }^{1, \S}$ Yu-Tang Wu, ${ }^{1}$ Paul M.P. van Bergen en Henegouwen, ${ }^{2}$

Travis L. Jennings, ${ }^{3}$ and Niko Hildebrandt ${ }^{1 *}$

1 NanoBioPhotonics (www.nanofret.com), Center for Nanoscience and Nanotechnology (C2N), Institute for Integrative Biology of the Cell (I2BC), Université Paris-Saclay, Université Paris-Sud, CNRS, CEA, 91405 Orsay Cedex, France

2 Cell Biology, Department of Biology, Science Faculty, Utrecht University, Utrecht, Netherlands.

3 Affymetrix, Inc., 10255 Science Center Drive, San Diego, California 92121, USA.

§ Current address: CEA, INAC-SyMMES, 17 rue des Martyrs, F-38054 Grenoble Cedex 9, France

* Corresponding author: niko.hildebrandt @u-psud.fr

\section{SUPPORTING INFORMATION}




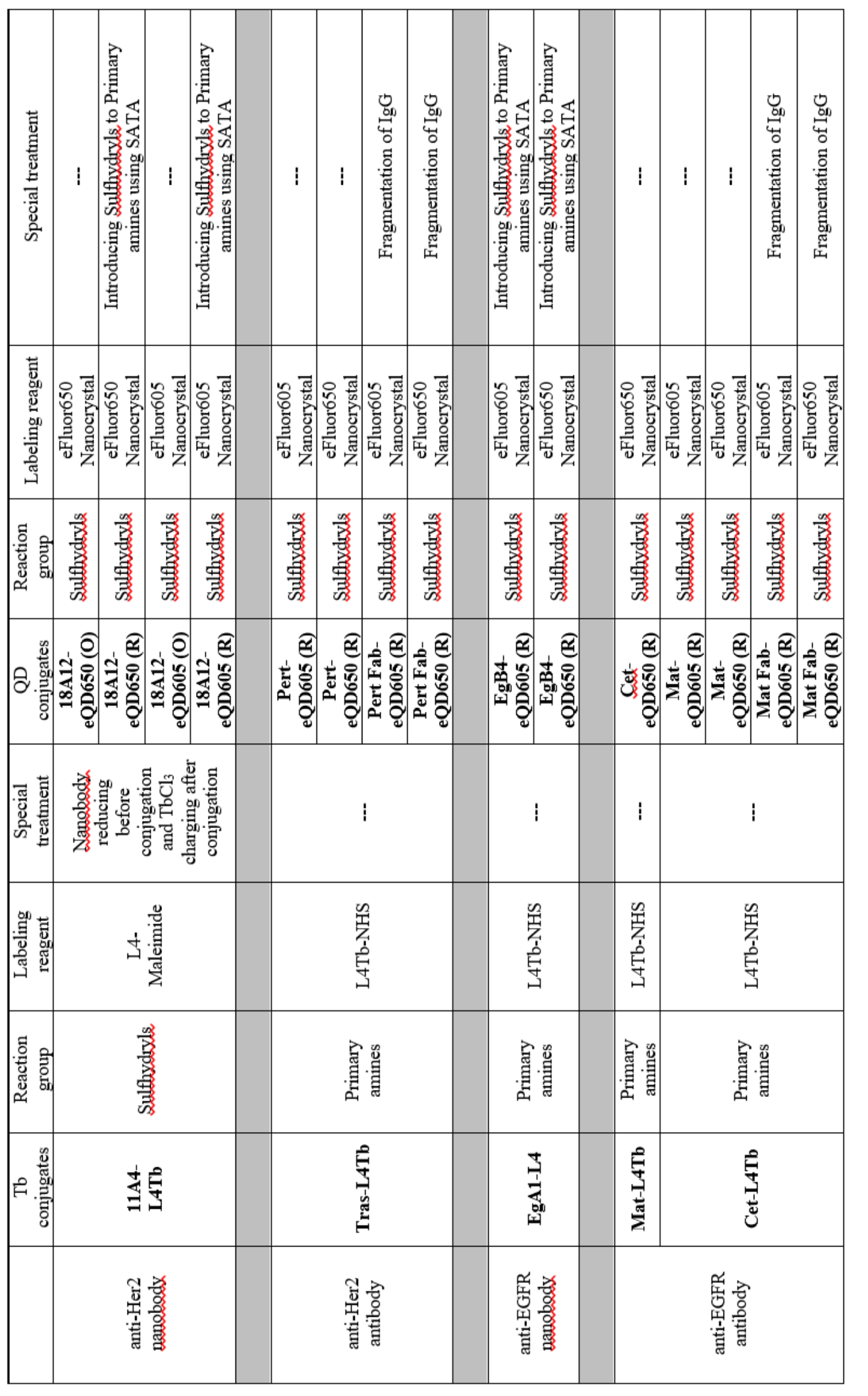

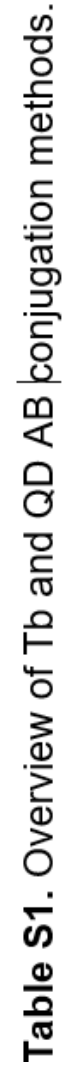



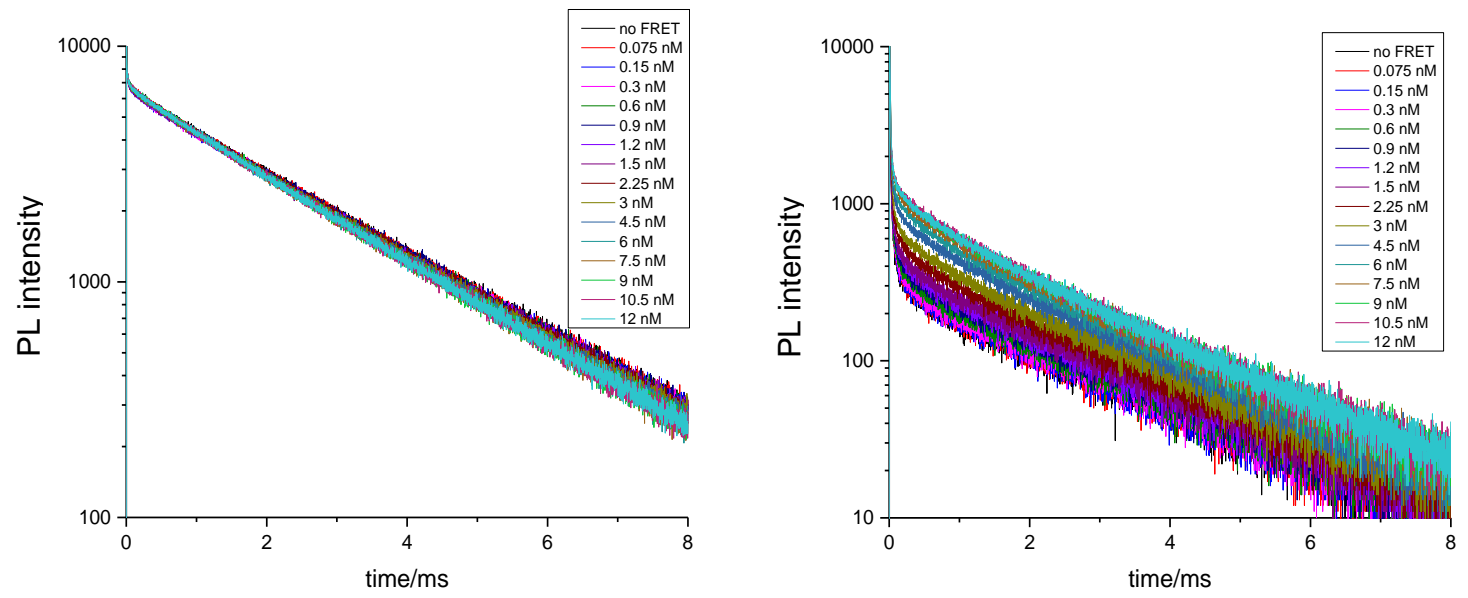

Figure S1: PL decay curves of Tb $(494 \pm 12 \mathrm{~nm}$, left), and QD650 (659 $\pm 10 \mathrm{~nm}$, right) of Tb-Mat-EGFR-CetQD650 immunoassays (in buffer)
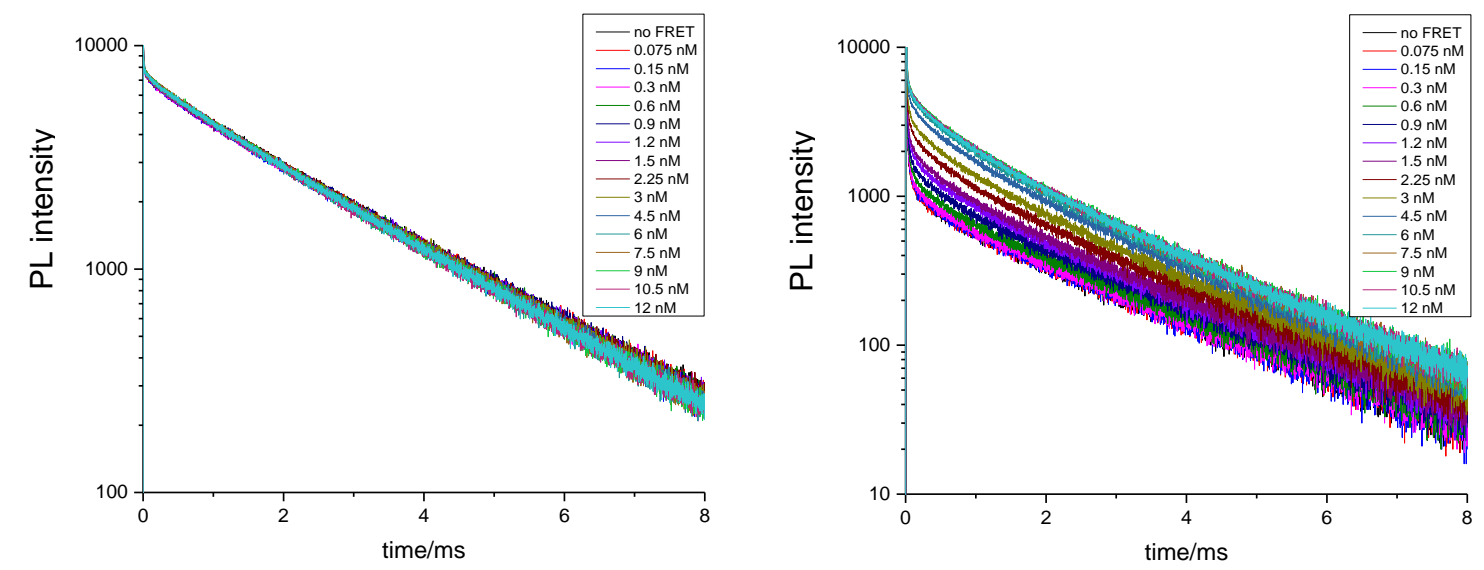

Figure S2: PL decay curves of Tb $(494 \pm 12 \mathrm{~nm}$, left), and QD650 (659 $\pm 10 \mathrm{~nm}$, right) of Tb-Cet-EGFR-MatQD650 immunoassays (in buffer)
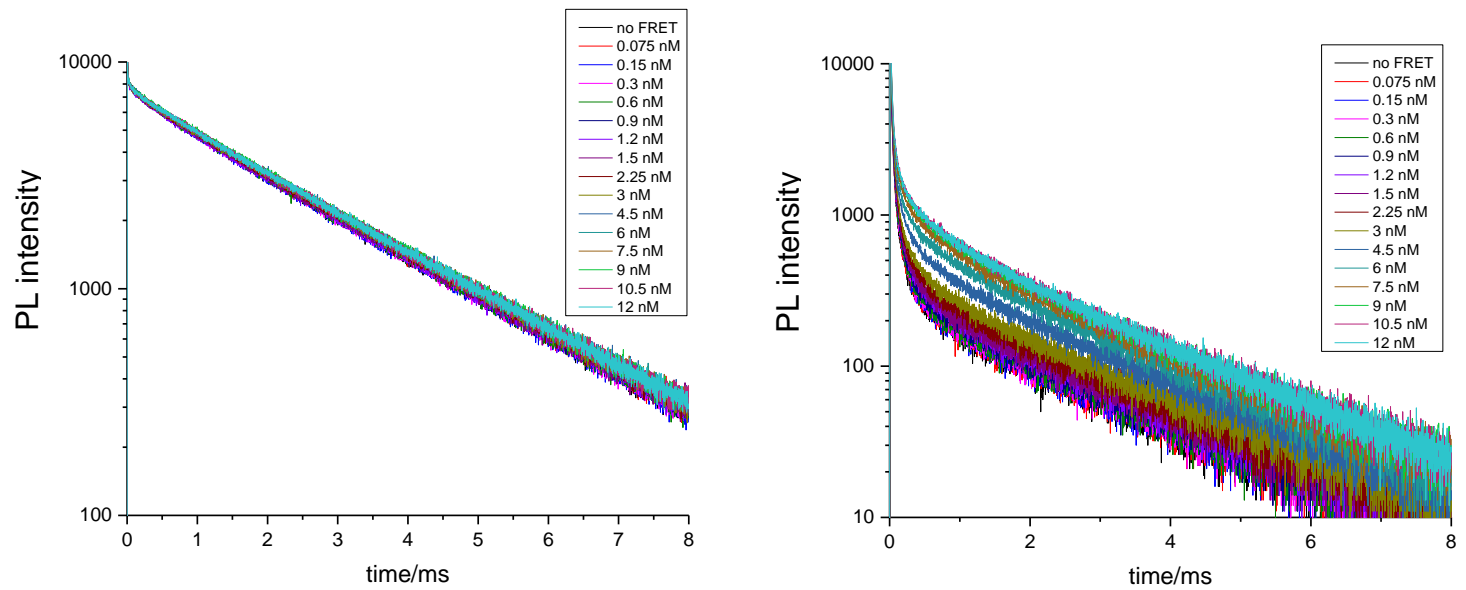

Figure S3: PL decay curves of Tb $(494 \pm 12 \mathrm{~nm}$, left), and QD605 (608 $\pm 4 \mathrm{~nm}$, right) of Tb-Cet-EGFR-MatQD605 immunoassays (in buffer) 

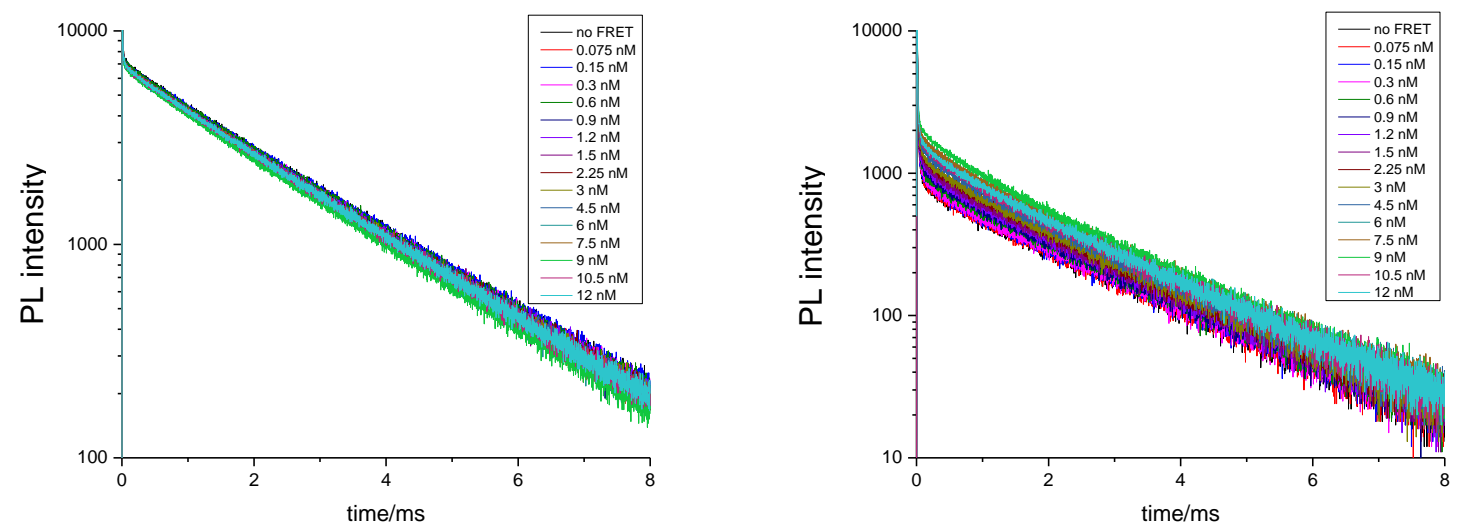

Figure S4: PL decay curves of Tb (494 $\pm 12 \mathrm{~nm}$, left), and QD650 (659 $\pm 10 \mathrm{~nm}$, right) of Tb-Cet-EGFR-MatQD650 immunoassays (in serum)
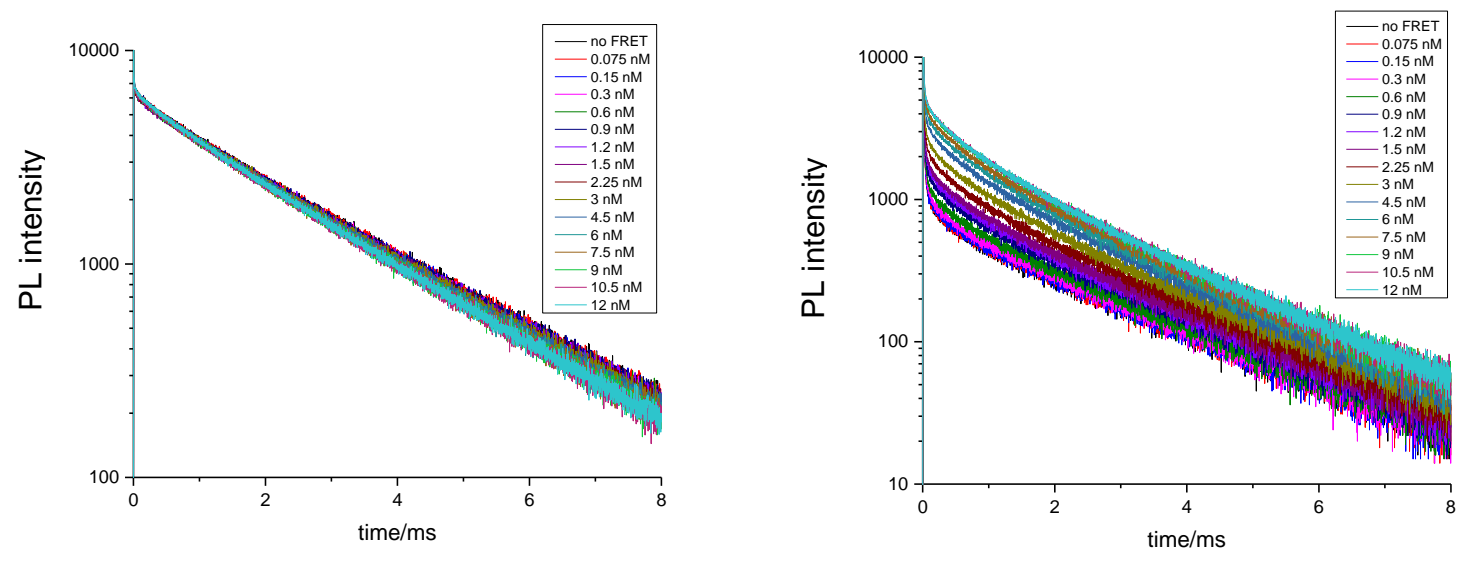

Figure S5: PL decay curves of Tb $(494 \pm 12 \mathrm{~nm}$, left), and QD650 (659 $\pm 10 \mathrm{~nm}$, right) of Tb-Cet-EGFRMatFab-QD650 immunoassays (in buffer)
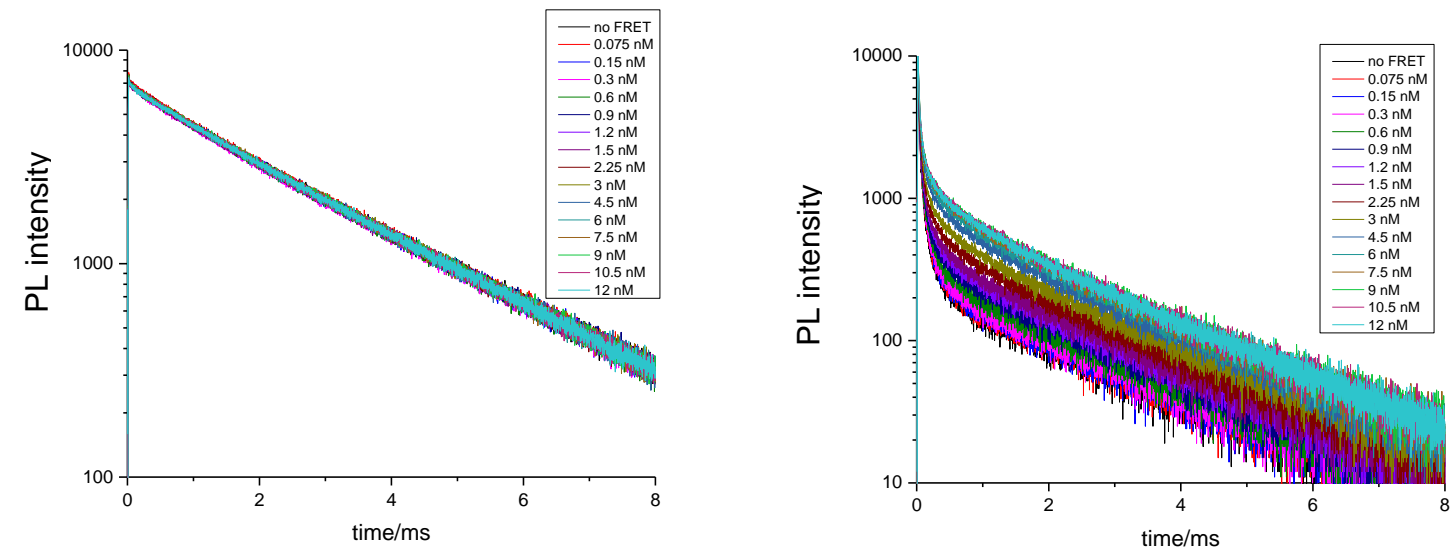

Figure S6: PL decay curves of Tb (494 $\pm 12 \mathrm{~nm}$, left), and QD605 (608 $\pm 4 \mathrm{~nm}$, right) of Tb-Cet-EGFR-MatFabQD605 immunoassays (in buffer) 

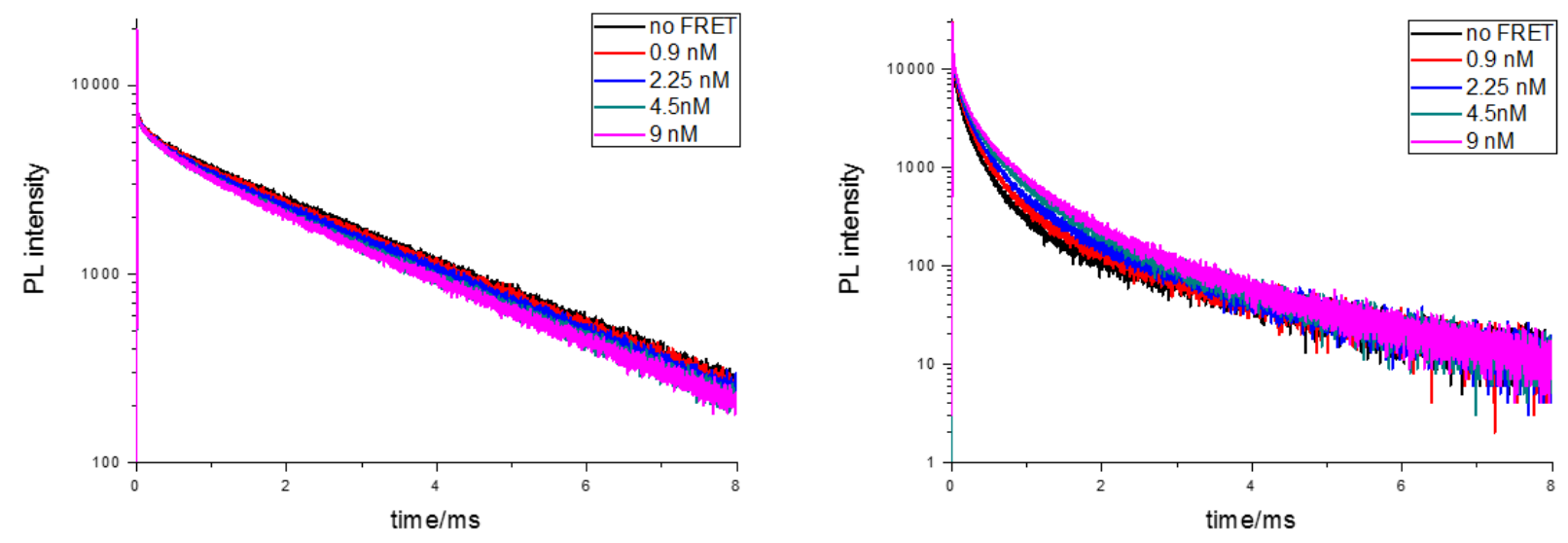

Figure S7: PL decay curves of Tb $(494 \pm 12 \mathrm{~nm}$, left), and QD650 (659 $\pm 10 \mathrm{~nm}$, right) of Tb-EgA1-EGFREgB4-QD650 immunoassays (in buffer)
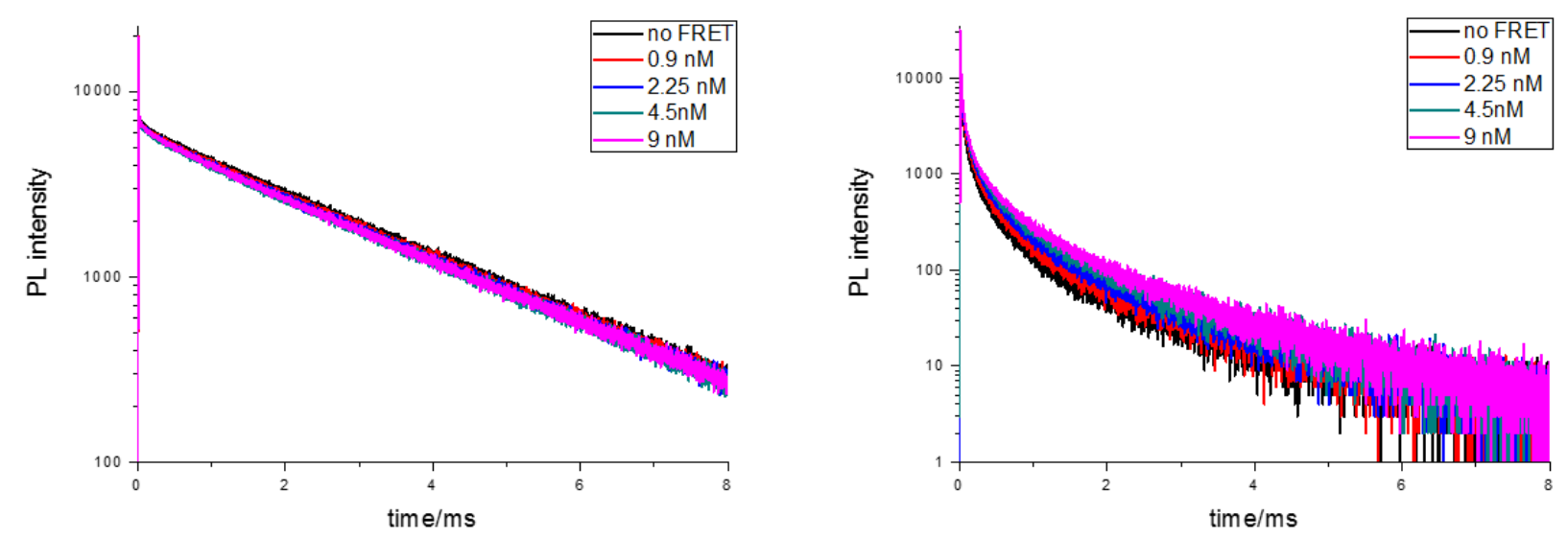

Figure S8: PL decay curves of Tb $(494 \pm 12 \mathrm{~nm}$, left), and QD605 (608 $\pm 4 \mathrm{~nm}$, right) of Tb-EgA1-EGFR-EgB4QD605 immunoassays (in buffer) 

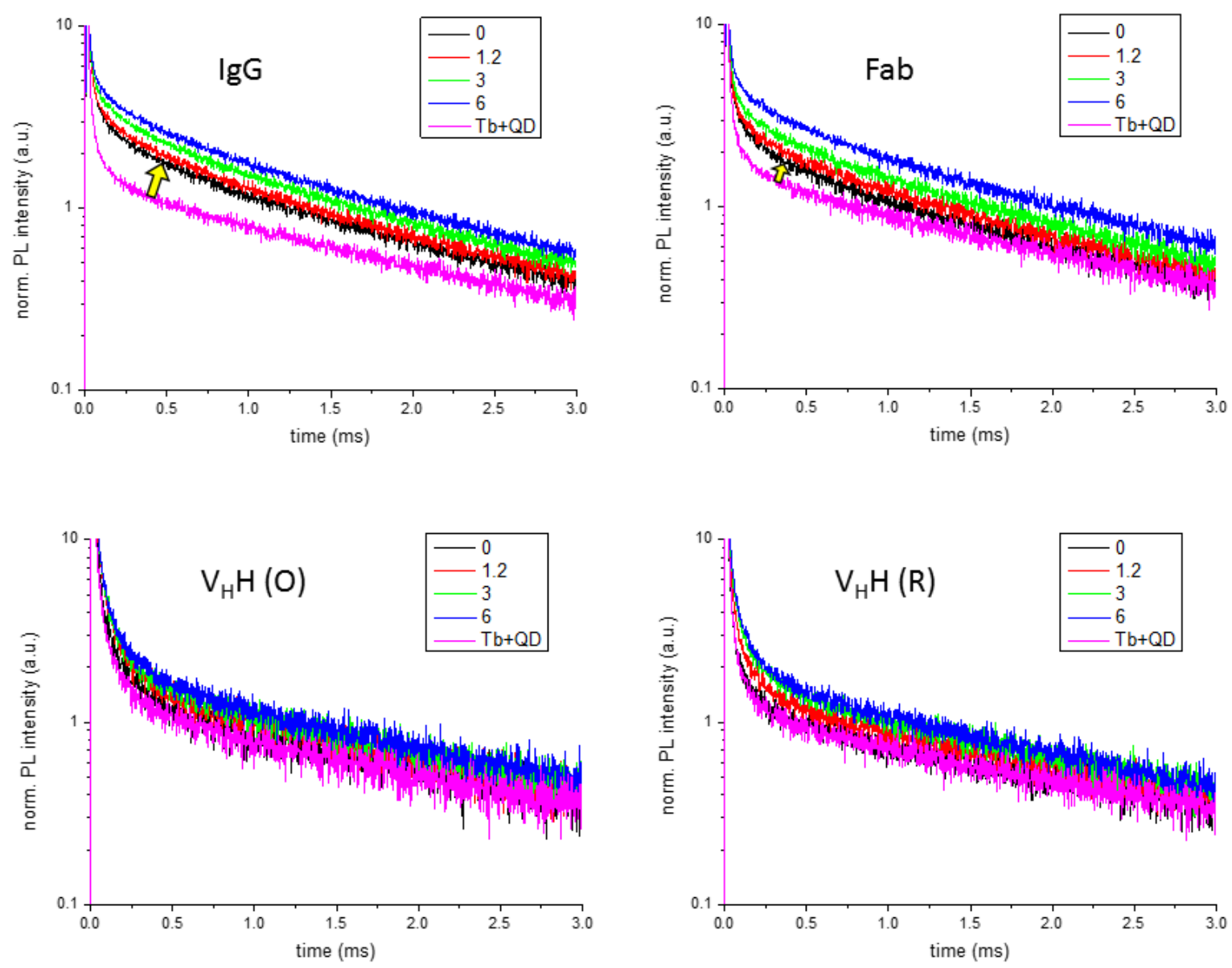

Figure S9: Intensity normalized QD650 PL decay curves of the $\mathrm{IgG}$, Fab, and $\mathrm{V}_{\mathrm{H}} \mathrm{H}$ based HER2 immunoassays with concentrations of 0 (black), 1.2 (red), 3 (green), and 6 (blue) nM compared to a curve that presents the mathematical sum of QD+Tb PL (magenta). IgG and Fab assays show significant FRET even without any HER2 in the sample, as visualized by the yellow arrows (difference between the sum of $\mathrm{Tb}+\mathrm{QD}$ and the $\mathrm{Tb}-\mathrm{AB}$ and $\mathrm{QD}-\mathrm{AB}$ sample in biological buffer). This pre-FRET is most probably caused by non-specific binding to BSA. 


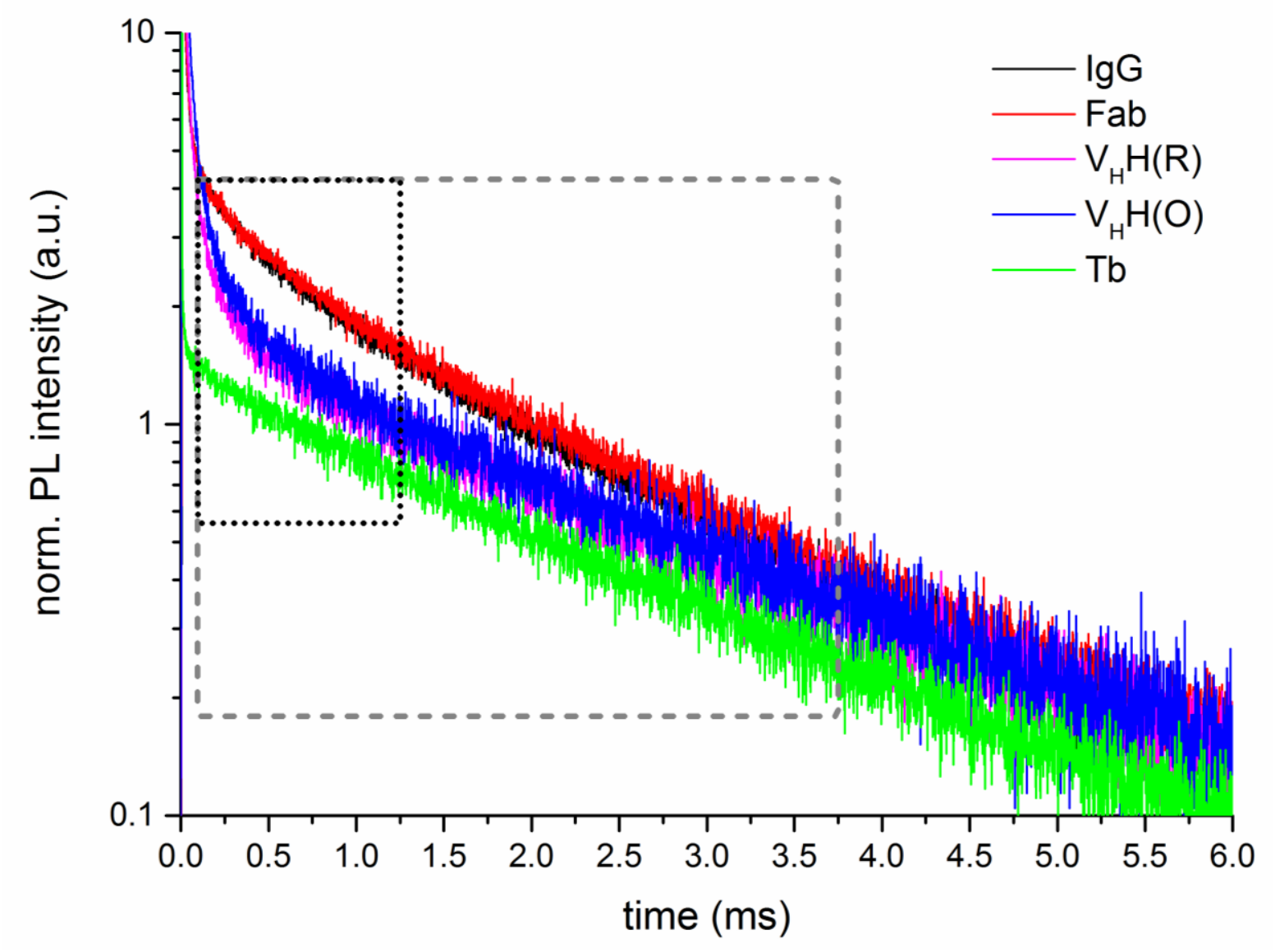

Figure S10: Intensity normalized (to the green curve at $0.5 \mathrm{~ms}$ ) QD650 PL decay curves of Tb-Tras-HER2Pert-QD650 (black), Tb-Tras-HER2-PertFab-QD650 (red), Tb-Cys11A4-HER2-18A12-QD650 (magenta), and Tb-Cys11A4-HER2-18A12cys-QD650 (blue) immunoassays and of pure Tb-AB (green). The IgG and Fab immunoassays show a much longer FRET decay (black and red within the gray dashed box) than the $\mathrm{V}_{\mathrm{H}} \mathrm{H}$ assays (blue and magenta within the black dotted box), which signifies a longer donor-acceptor distance in the case of $\lg \mathrm{G}$ and $\mathrm{Fab}$ compared to $\mathrm{V}_{\mathrm{H}} \mathrm{H}$. 

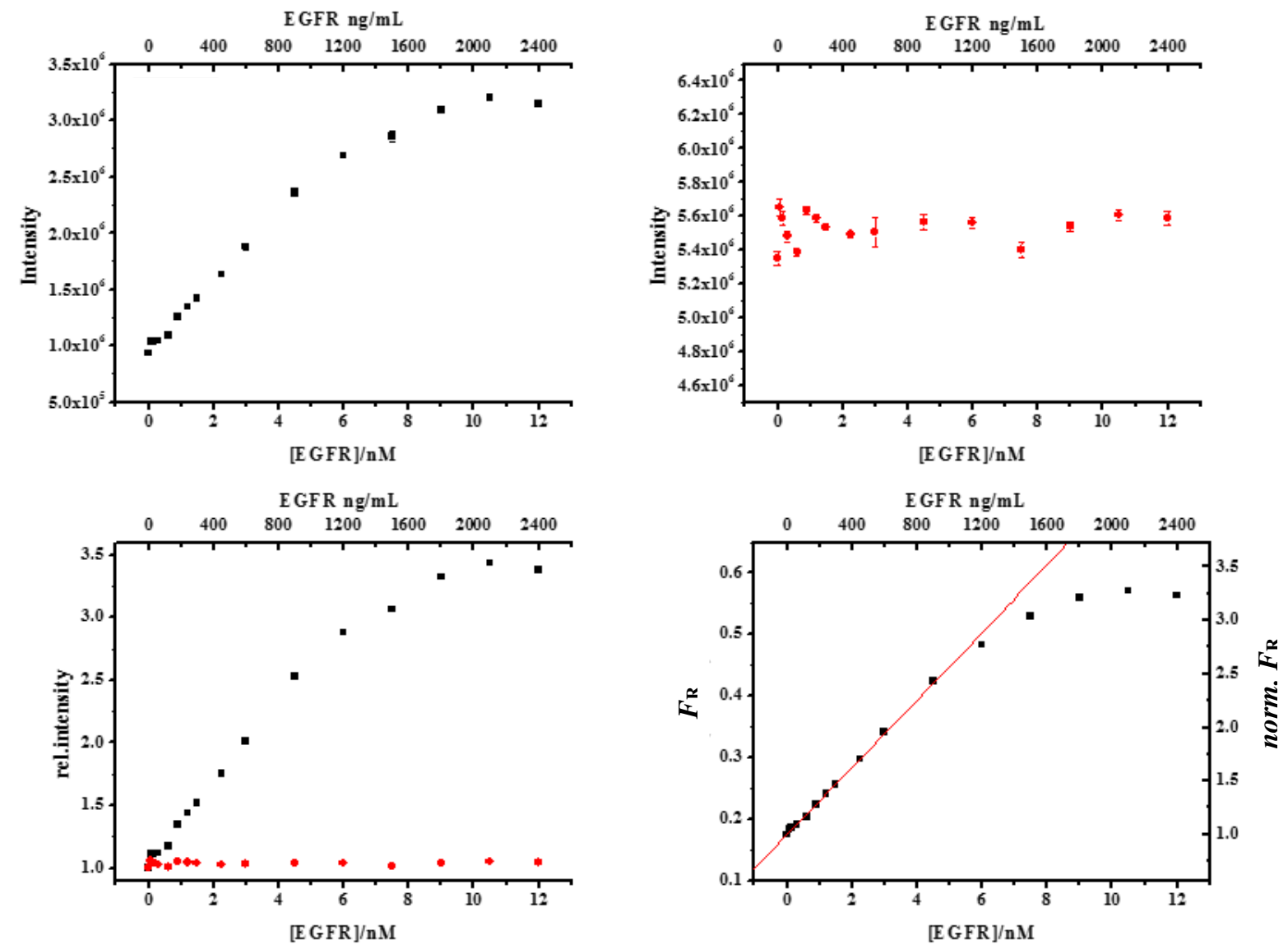

Figure S11: Tb-Mat-EGFR-Cet-QD650 immunoassay (in buffer). Top left: Time-gated (0.1 - $0.9 \mathrm{~ms}) \mathrm{PL}$ intensity in the QD channel over EGFR concentration. Top right: Time-gated $(0.1-0.9 \mathrm{~ms}) \mathrm{PL}$ intensity in the Tb channel over EGFR concentration. Bottom left: Both time-gated intensities normalized to unity at zero EGFR. Bottom right: Linear part of the calibration curve that was used for calculating the LOD. 

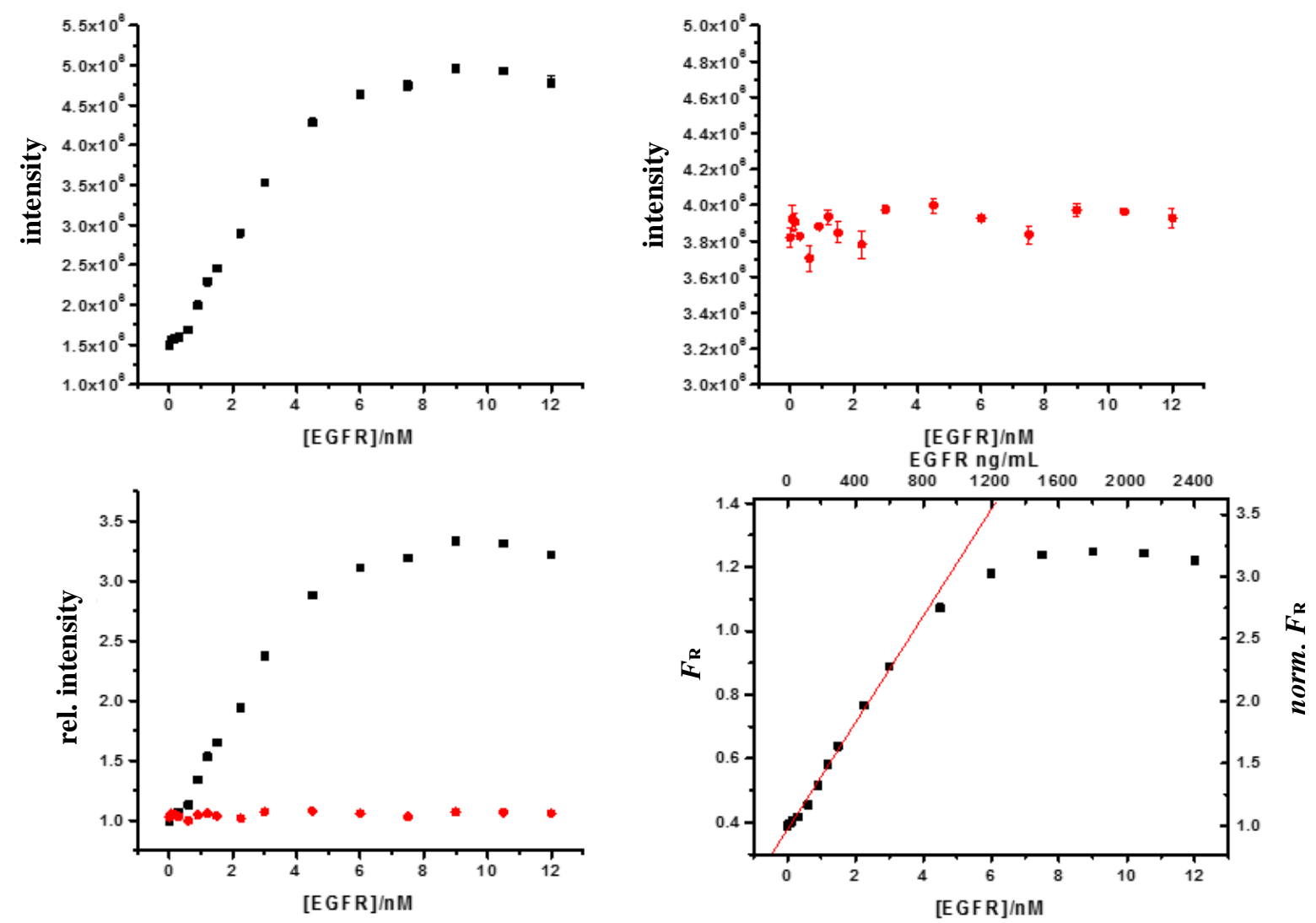

Figure S12: Tb-Cet-EGFR-Mat-QD650 immunoassays (in buffer): Top left: Time-gated $(0.1-0.9 \mathrm{~ms}) \mathrm{PL}$ intensity in the QD channel over EGFR concentration. Top right: Time-gated $(0.1-0.9 \mathrm{~ms}) \mathrm{PL}$ intensity in the Tb channel over EGFR concentration. Bottom left: Both time-gated intensities normalized to unity at zero EGFR. Bottom right: Linear part of the calibration curve that was used for calculating the LOD. 

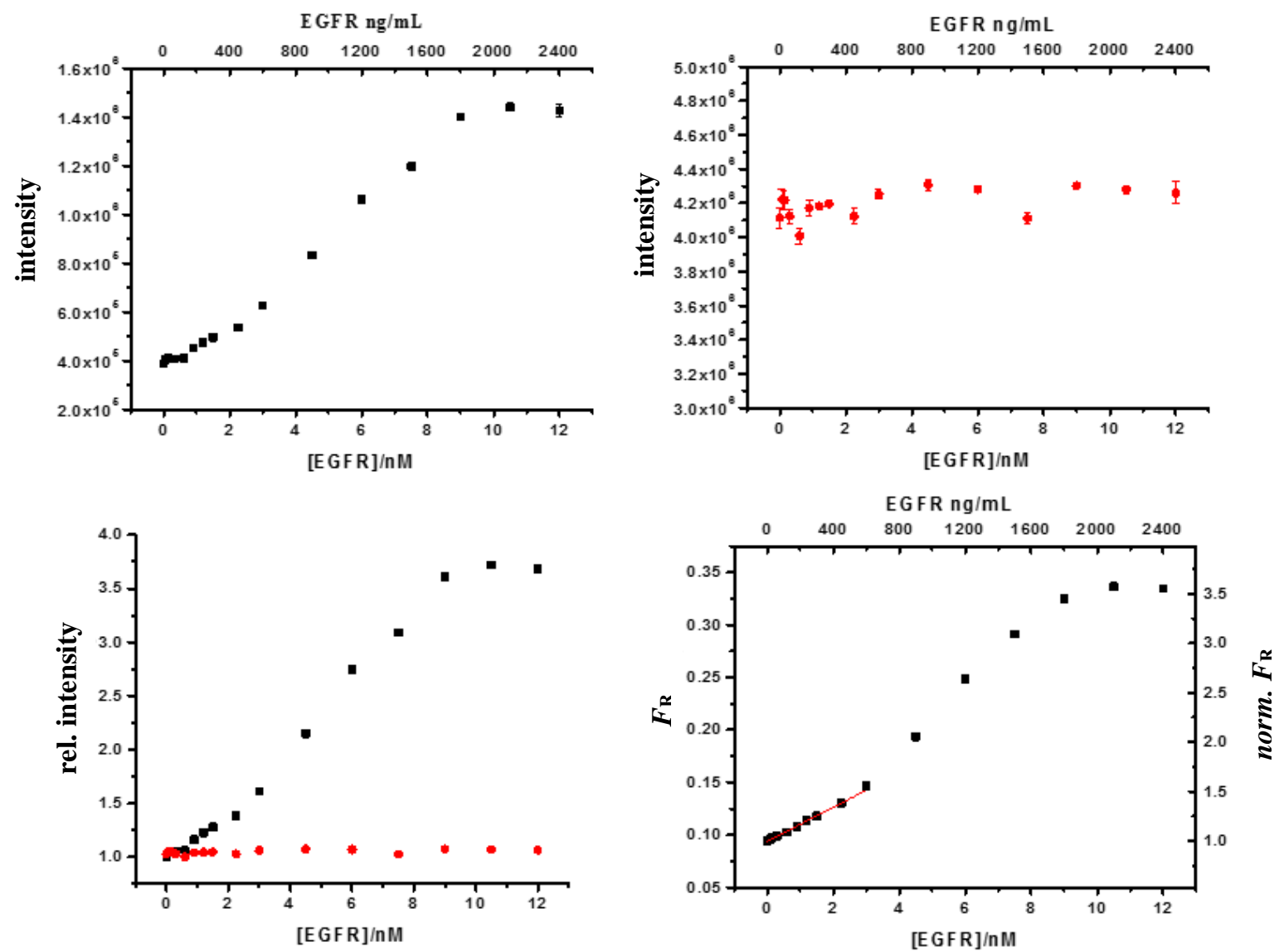

Figure S13: Tb-Cet-EGFR-Mat-QD605 immunoassays (in buffer): Top left: Time-gated (0.1 - $0.9 \mathrm{~ms}) \mathrm{PL}$ intensity in the QD channel over EGFR concentration. Top right: Time-gated $(0.1-0.9 \mathrm{~ms}) \mathrm{PL}$ intensity in the Tb channel over EGFR concentration. Bottom left: Both time-gated intensities normalized to unity at zero EGFR. Bottom right: Linear part of the calibration curve that was used for calculating the LOD. 

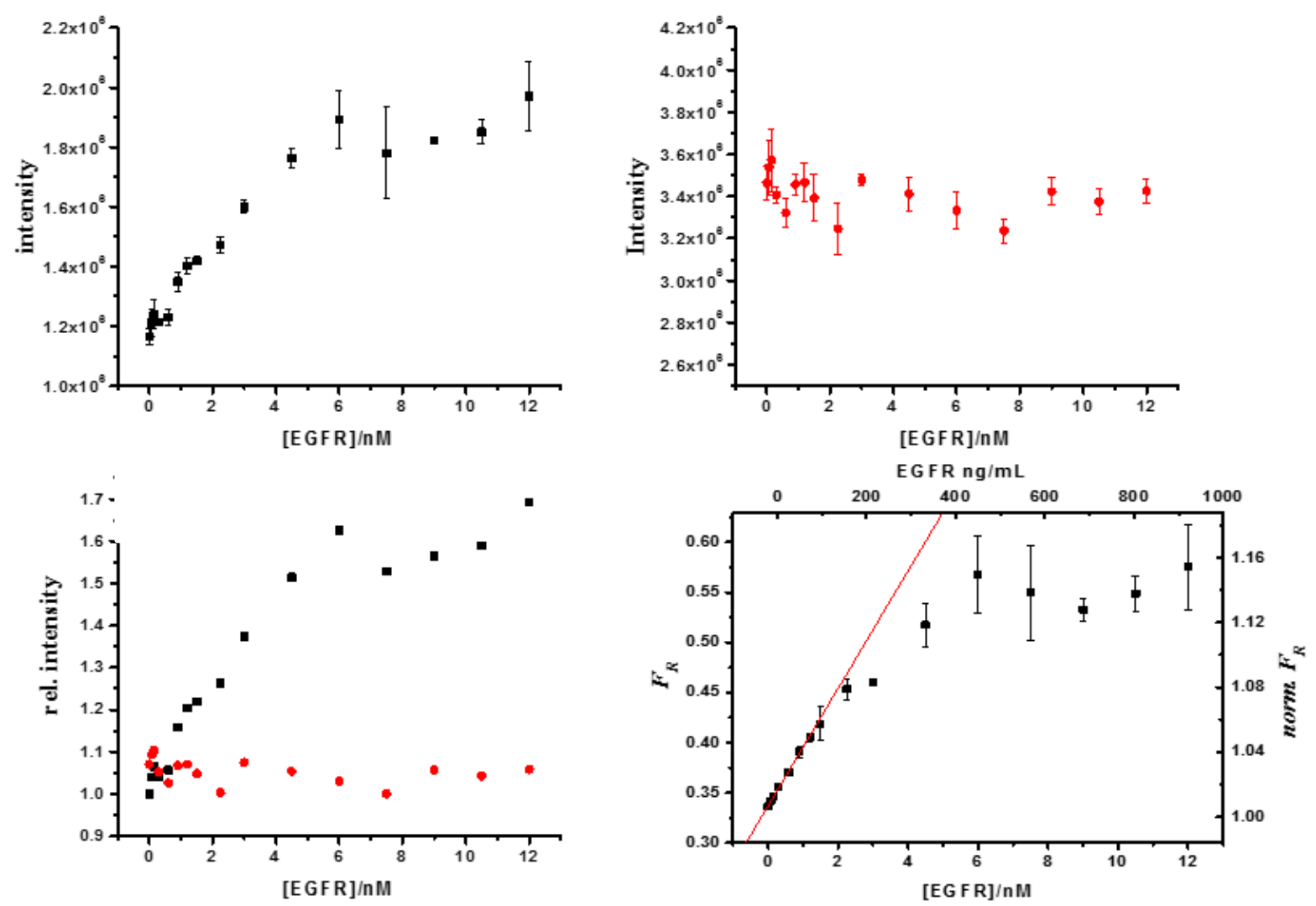

Figure S14: Tb-Cet-EGFR-Mat-QD650 immunoassays (in serum): Top left: Time-gated $(0.1-0.9 \mathrm{~ms}) \mathrm{PL}$ intensity in the QD channel over EGFR concentration. Top right: Time-gated $(0.1-0.9 \mathrm{~ms}) \mathrm{PL}$ intensity in the Tb channel over EGFR concentration. Bottom left: Both time-gated intensities normalized to unity at zero EGFR. Bottom right: Linear part of the calibration curve that was used for calculating the LOD. 

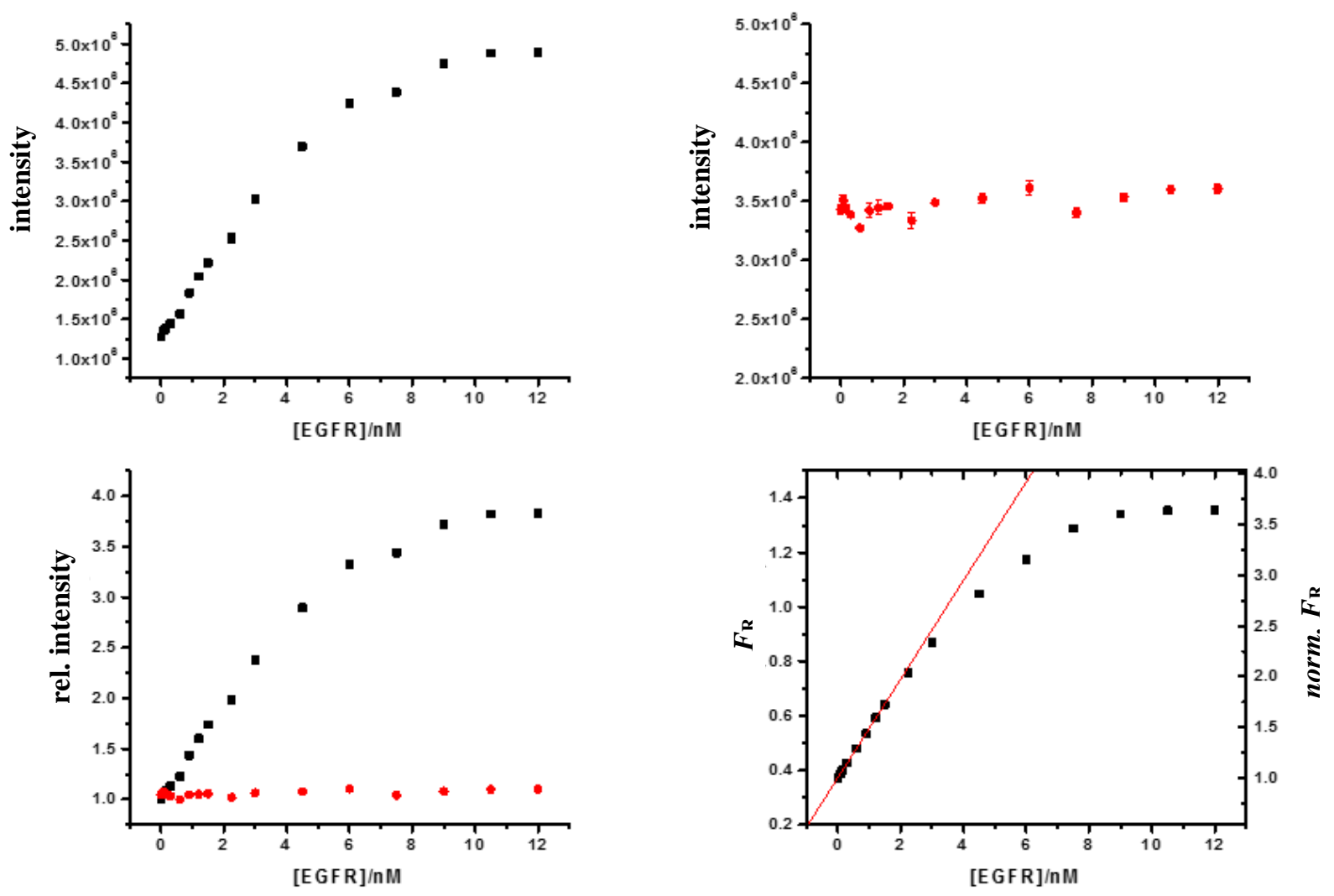

Figure S15: Tb-Cet-EGFR-MatFab-QD650 immunoassays (in buffer): Top left: Time-gated (0.1- $0.9 \mathrm{~ms})$ PL intensity in the QD channel over EGFR concentration. Top right: Time-gated $(0.1-0.9 \mathrm{~ms}) \mathrm{PL}$ intensity in the Tb channel over EGFR concentration. Bottom left: Both time-gated intensities normalized to unity at zero EGFR. Bottom right: Linear part of the calibration curve that was used for calculating the LOD. 

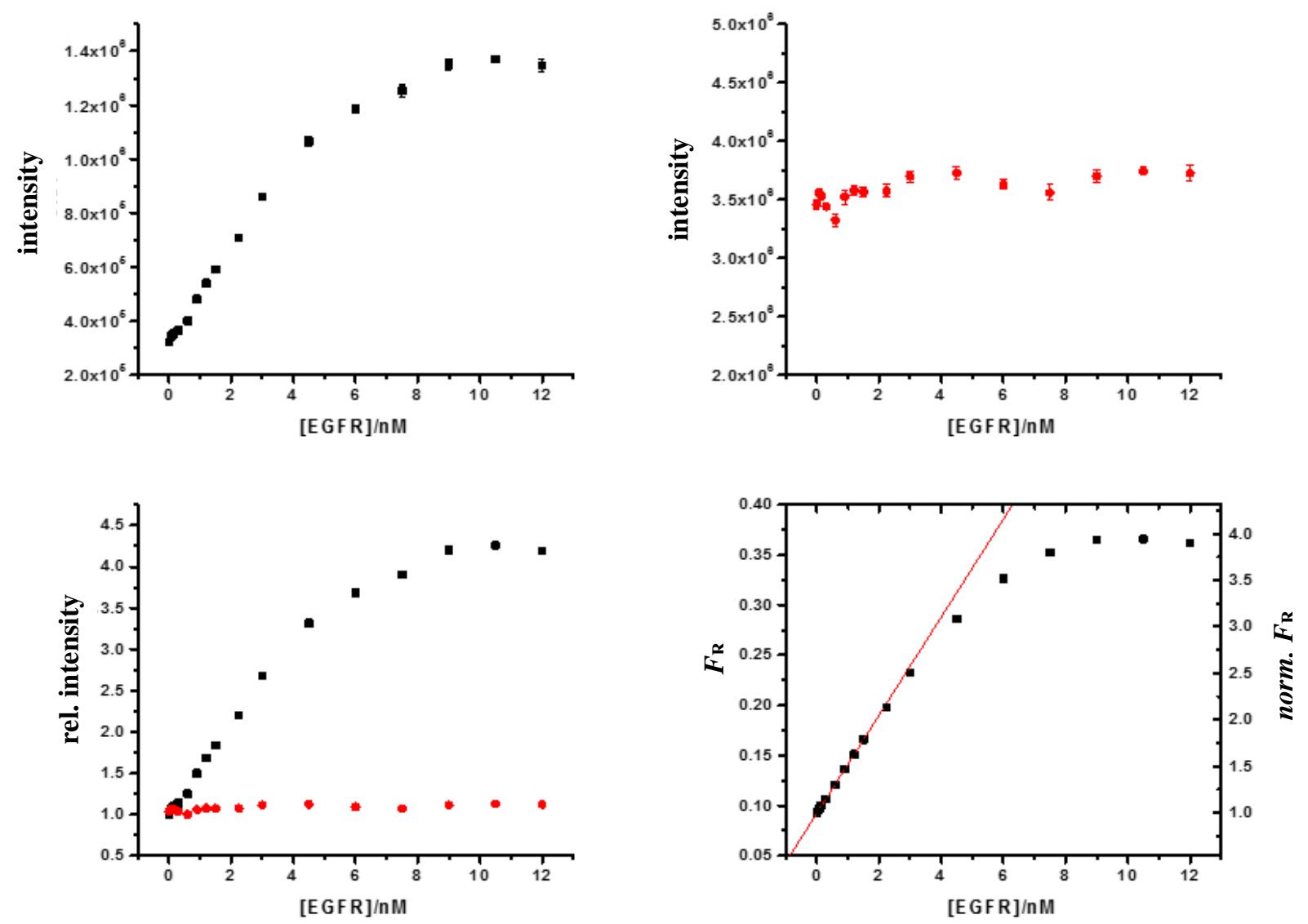

Figure S16: Tb-Cet-EGFR-MatFab-QD605 immunoassays (in buffer): Top left: Time-gated (0.1 - $0.9 \mathrm{~ms})$ PL intensity in the QD channel over EGFR concentration. Top right: Time-gated $(0.1-0.9 \mathrm{~ms}) \mathrm{PL}$ intensity in the Tb channel over EGFR concentration. Bottom left: Both time-gated intensities normalized to unity at zero EGFR. Bottom right: Linear part of the calibration curve that was used for calculating the LOD. 

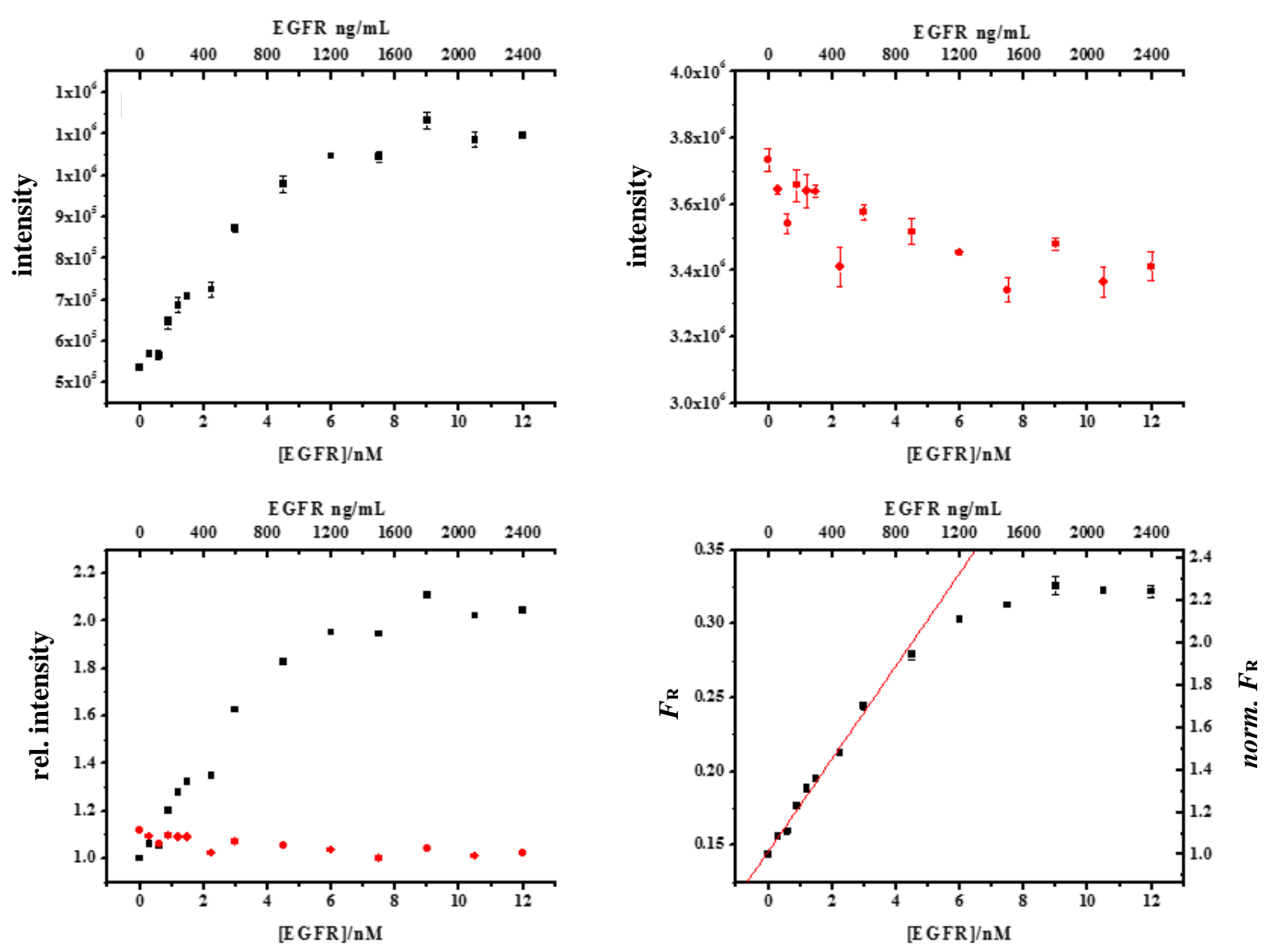

Figure S17: Tb-EgA1-EGFR-EgB4-QD650 immunoassays (in buffer): Top left: Time-gated (0.1 - $0.9 \mathrm{~ms})$ PL intensity in the QD channel over EGFR concentration. Top right: Time-gated $(0.1-0.9 \mathrm{~ms}) \mathrm{PL}$ intensity in the Tb channel over EGFR concentration. Bottom left: Both time-gated intensities normalized to unity at zero EGFR. Bottom right: Linear part of the calibration curve that was used for calculating the LOD. 

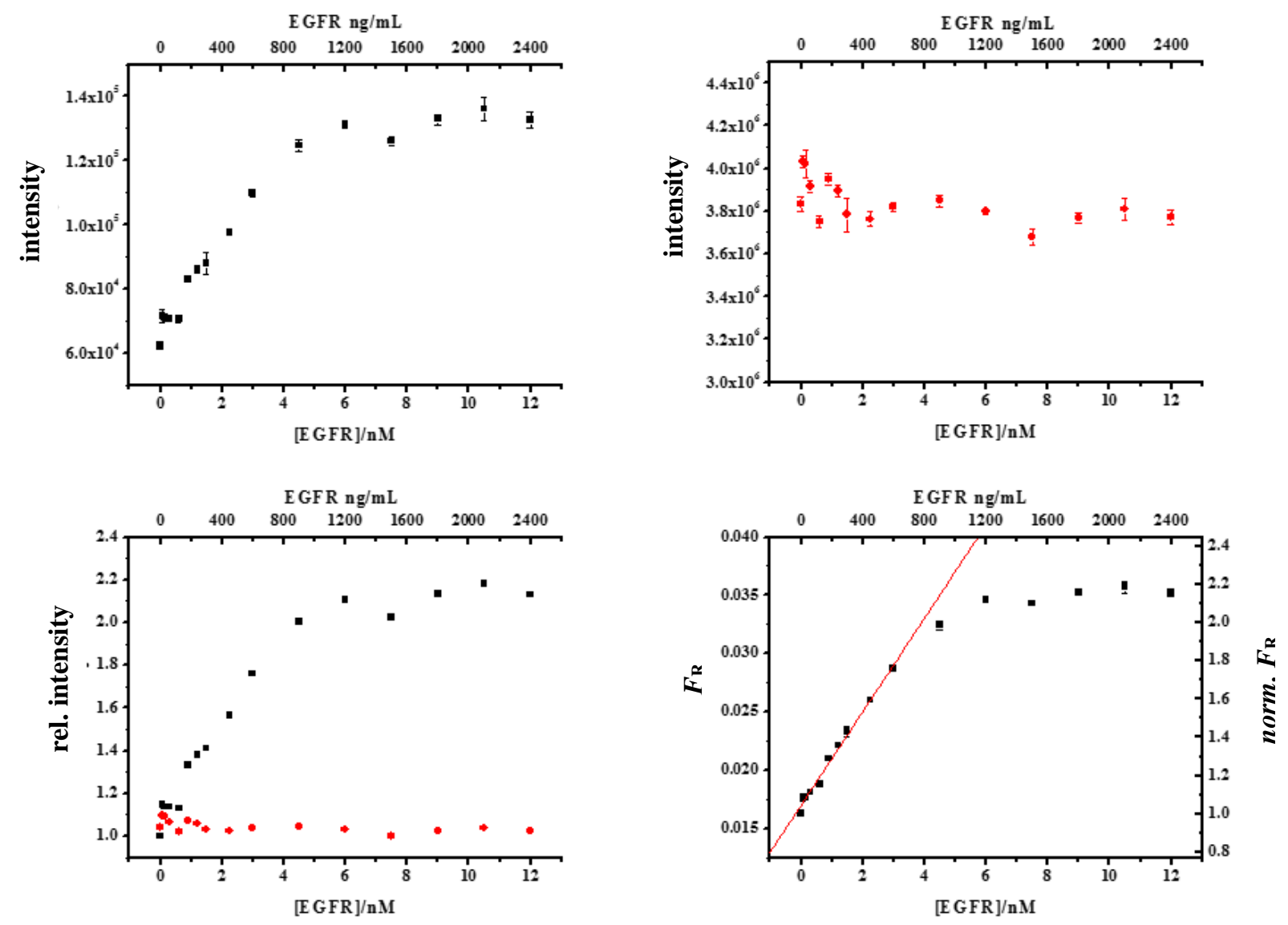

Figure S18: Tb-EgA1-EGFR-EgB4-QD605 immunoassays (in buffer): Top left: Time-gated (0.1 - $0.9 \mathrm{~ms})$ PL intensity in the QD channel over EGFR concentration. Top right: Time-gated $(0.1-0.9 \mathrm{~ms}) \mathrm{PL}$ intensity in the Tb channel over EGFR concentration. Bottom left: Both time-gated intensities normalized to unity at zero EGFR. Bottom right: Linear part of the calibration curve that was used for calculating the LOD. 

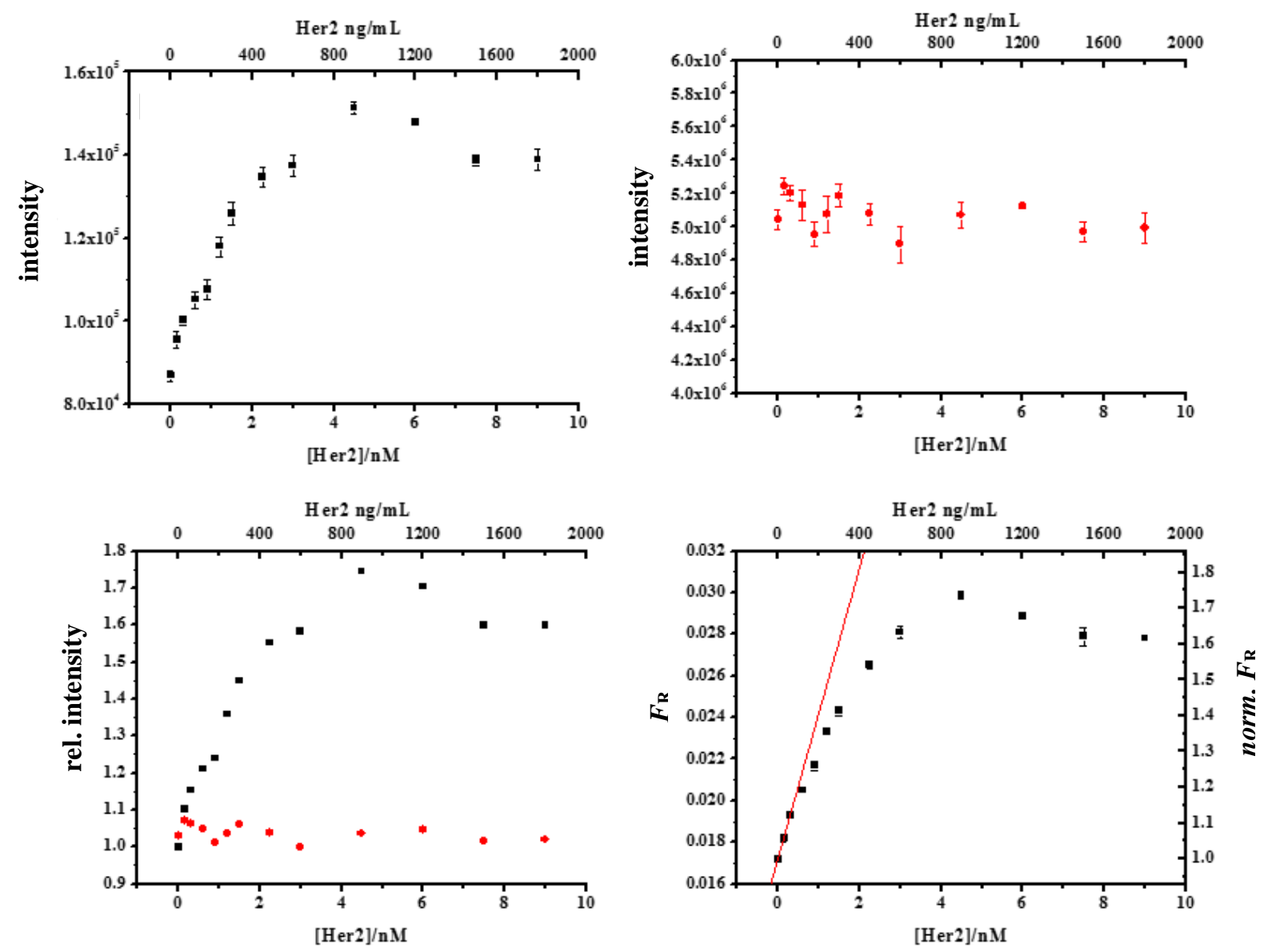

Figure S19: Tb-Cys11A4-HER2-18A12Cys-QD605 immunoassay (oriented conjugation). Top left: Timegated $(0.1-0.9 \mathrm{~ms}) \mathrm{PL}$ intensity in the QD channel over HER2 concentration. Top right: Time-gated $(0.1$ $-0.9 \mathrm{~ms}$ ) PL intensity in the Tb channel over HER2 concentration. Bottom left: Both time-gated intensities normalized to unity at zero HER2. Bottom right: Linear part of the calibration curve that was used for calculating the LOD. 

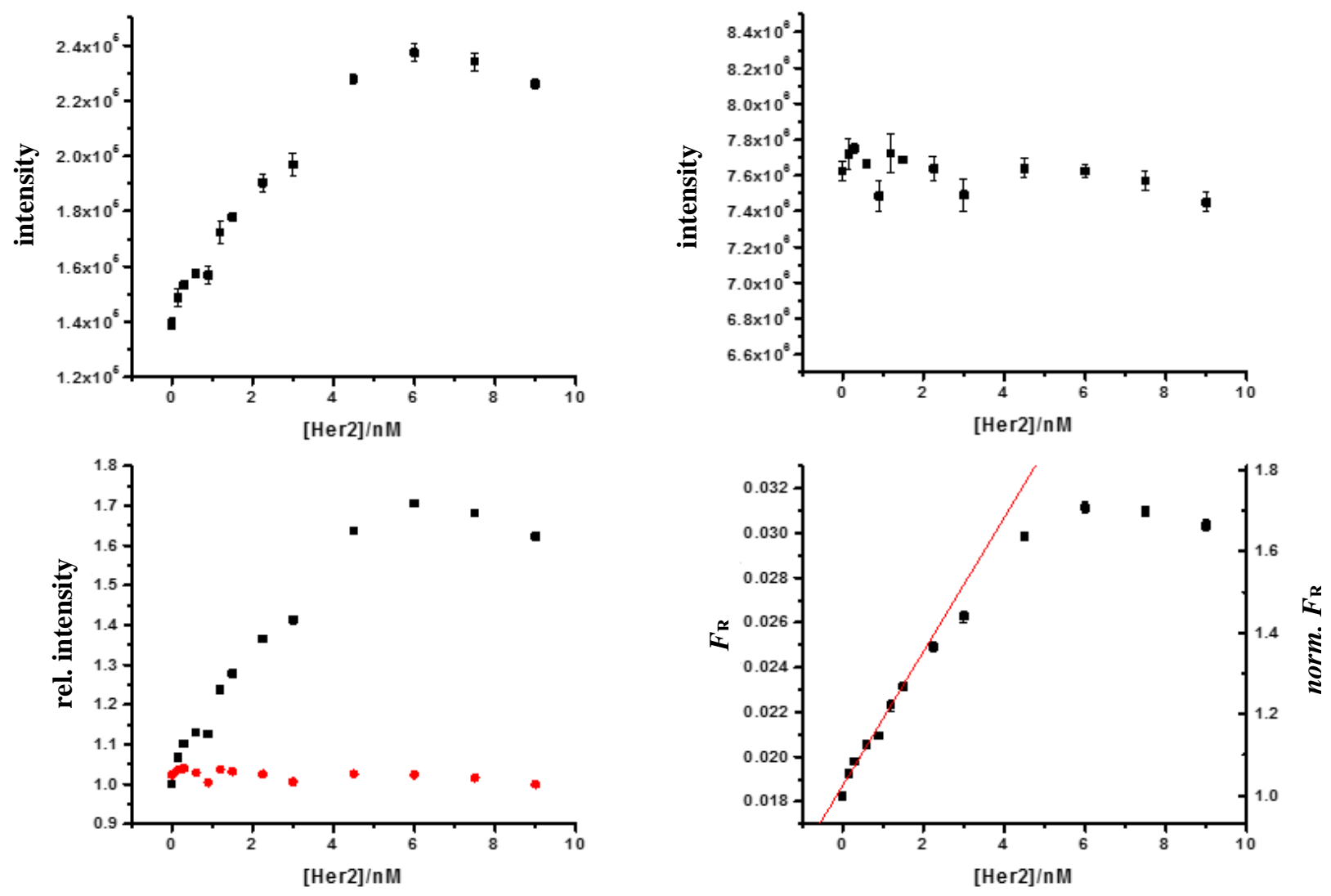

Figure S20: Tb-Cys11A4-HER2-18A12-QD605 immunoassay (random conjugation). Top left: Time-gated $(0.1-0.9 \mathrm{~ms}) \mathrm{PL}$ intensity in the QD channel over HER2 concentration. Top right: Time-gated $(0.1-0.9$ ms) PL intensity in the Tb channel over HER2 concentration. Bottom left: Both time-gated intensities normalized to unity at zero HER2. Bottom right: Linear part of the calibration curve that was used for calculating the LOD. 

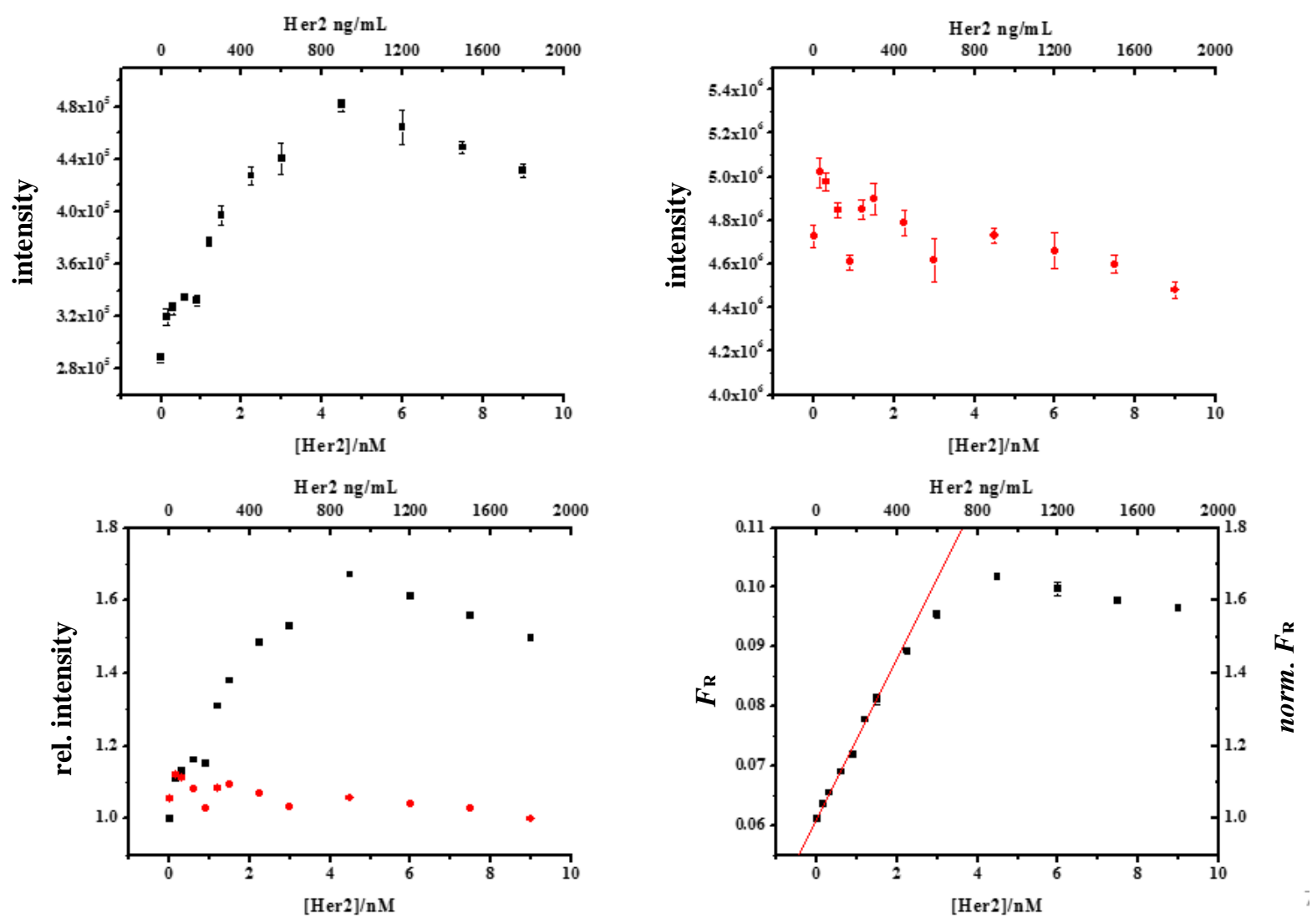

Figure S21: Tb-Cys11A4-HER2-18A12Cys-QD650 immunoassay (oriented conjugation). Top left: Timegated $(0.1-0.9 \mathrm{~ms}) \mathrm{PL}$ intensity in the QD channel over HER2 concentration. Top right: Time-gated $(0.1$ $-0.9 \mathrm{~ms}$ ) PL intensity in the Tb channel over HER2 concentration. Bottom left: Both time-gated intensities normalized to unity at zero HER2. Bottom right: Linear part of the calibration curve that was used for calculating the LOD. 

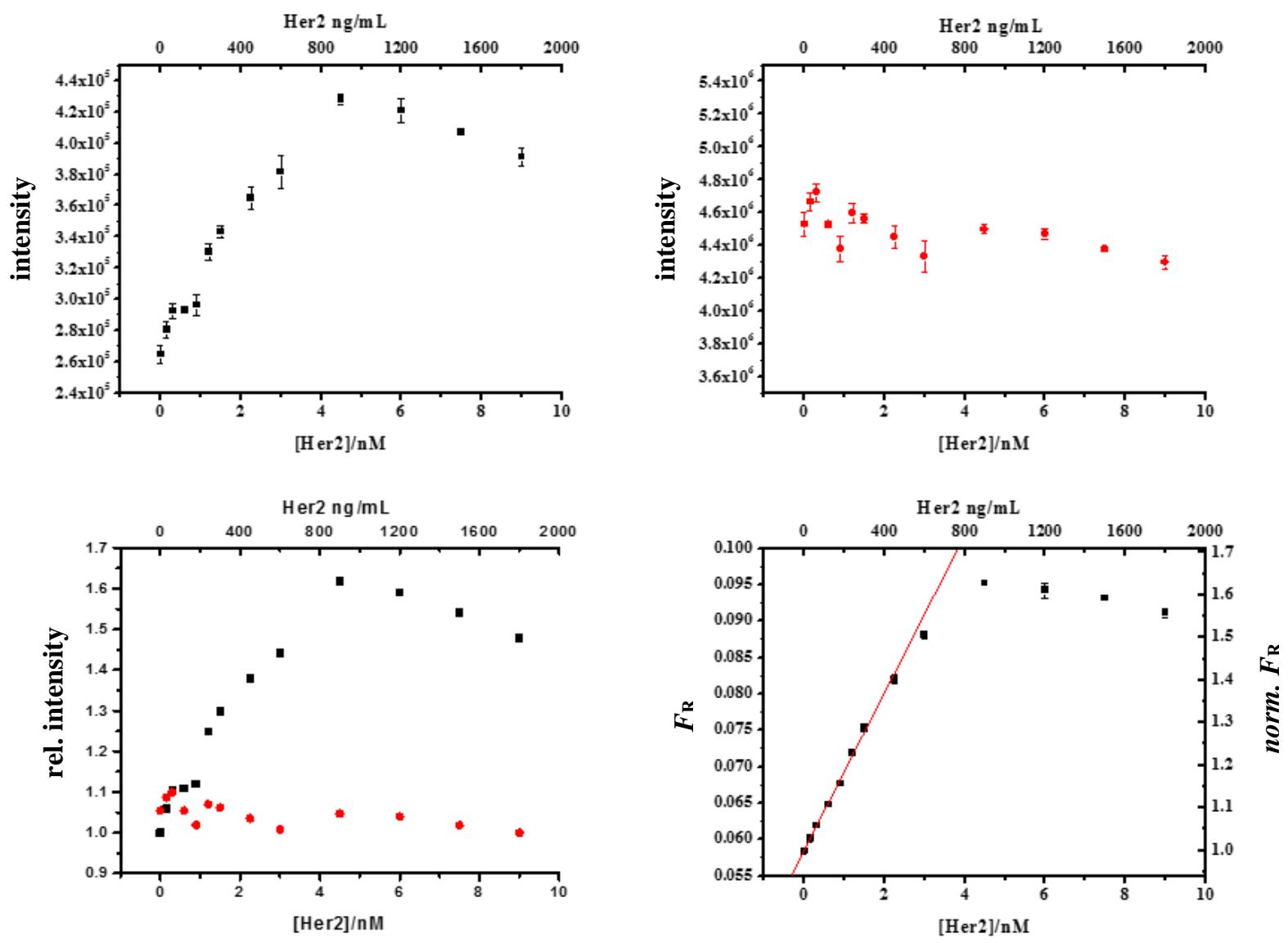

Figure S22: Tb-Cys11A4-HER2-18A12-QD650 immunoassay (random conjugation). Top left: Time-gated $(0.1-0.9 \mathrm{~ms}) \mathrm{PL}$ intensity in the QD channel over HER2 concentration. Top right: Time-gated $(0.1-0.9$ $\mathrm{ms}) \mathrm{PL}$ intensity in the Tb channel over HER2 concentration. Bottom left: Both time-gated intensities normalized to unity at zero HER2. Bottom right: Linear part of the calibration curve that was used for calculating the LOD. 

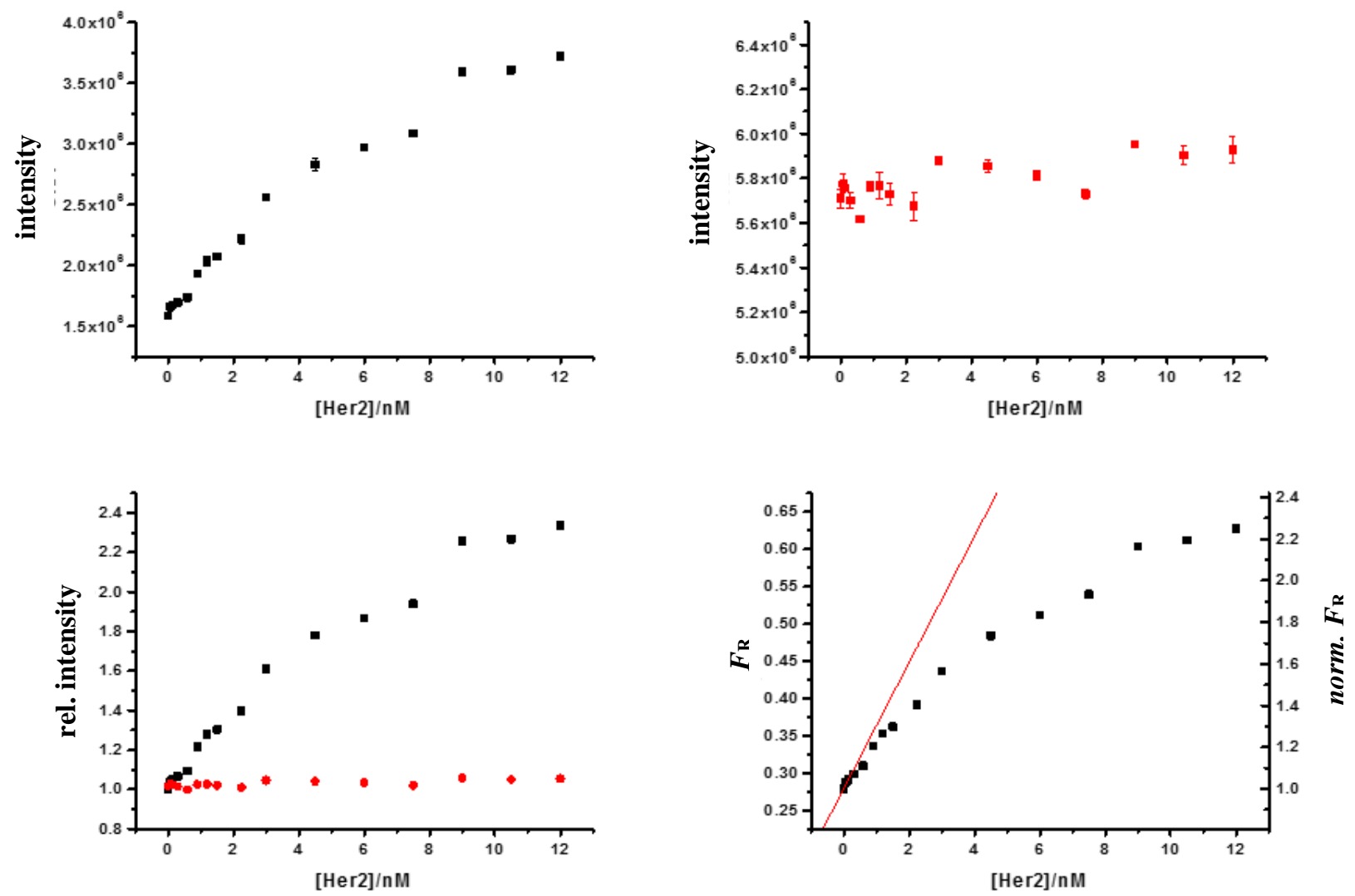

Figure S23: Tb-Tras-HER2-Pert-QD650 immunoassay. Top left: Time-gated (0.1-0.9 ms) PL intensity in the QD channel over HER2 concentration. Top right: Time-gated $(0.1-0.9 \mathrm{~ms}) \mathrm{PL}$ intensity in the Tb channel over HER2 concentration. Bottom left: Both time-gated intensities normalized to unity at zero HER2. Bottom right: Linear part of the calibration curve that was used for calculating the LOD. 

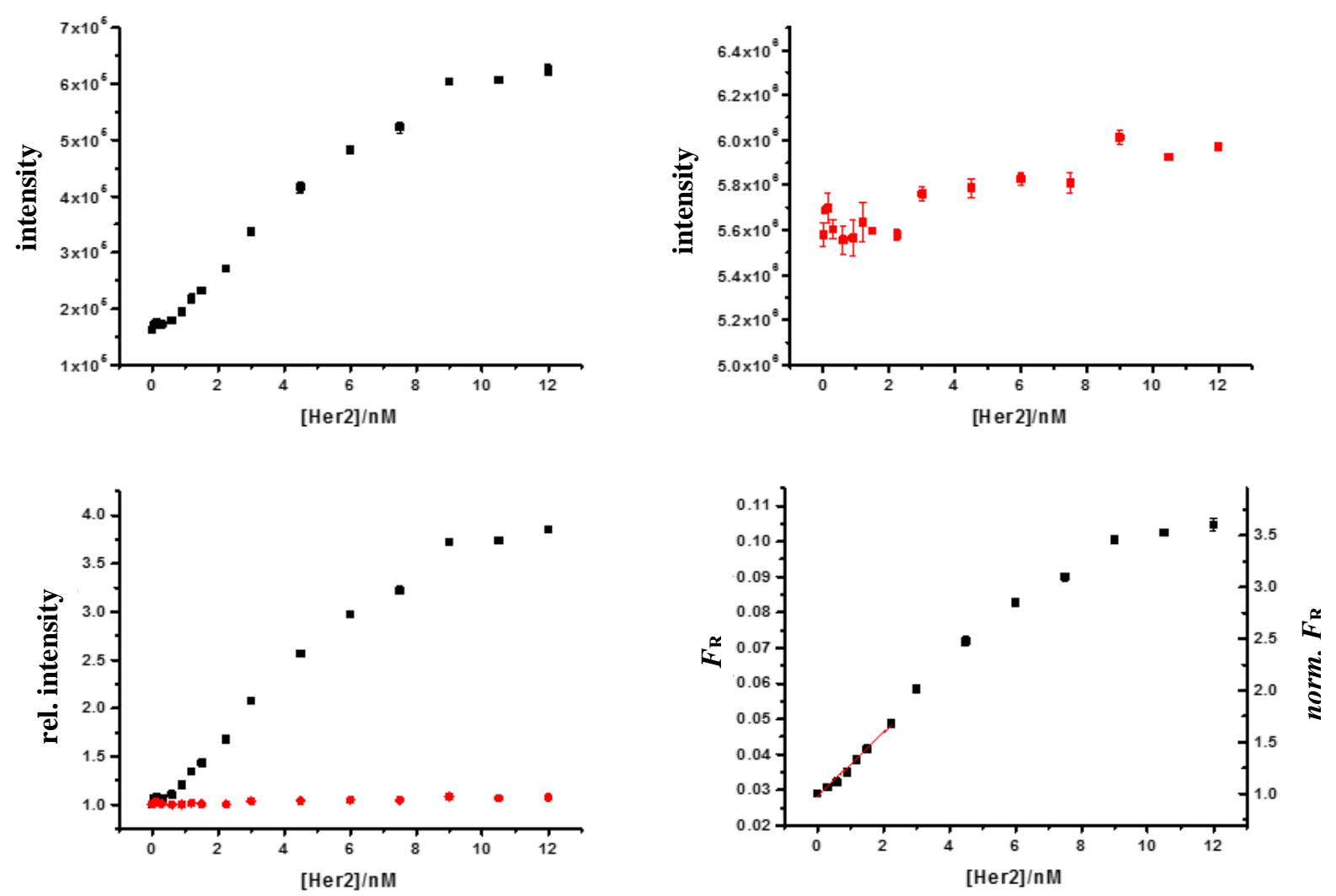

Figure S24: Tb-Tras-HER2-Pert-QD605 immunoassay. Top left: Time-gated (0.1 $-0.9 \mathrm{~ms}) \mathrm{PL}$ intensity in the QD channel over HER2 concentration. Top right: Time-gated $(0.1-0.9 \mathrm{~ms}) \mathrm{PL}$ intensity in the Tb channel over HER2 concentration. Bottom left: Both time-gated intensities normalized to unity at zero HER2. Bottom right: Linear part of the calibration curve that was used for calculating the LOD. 

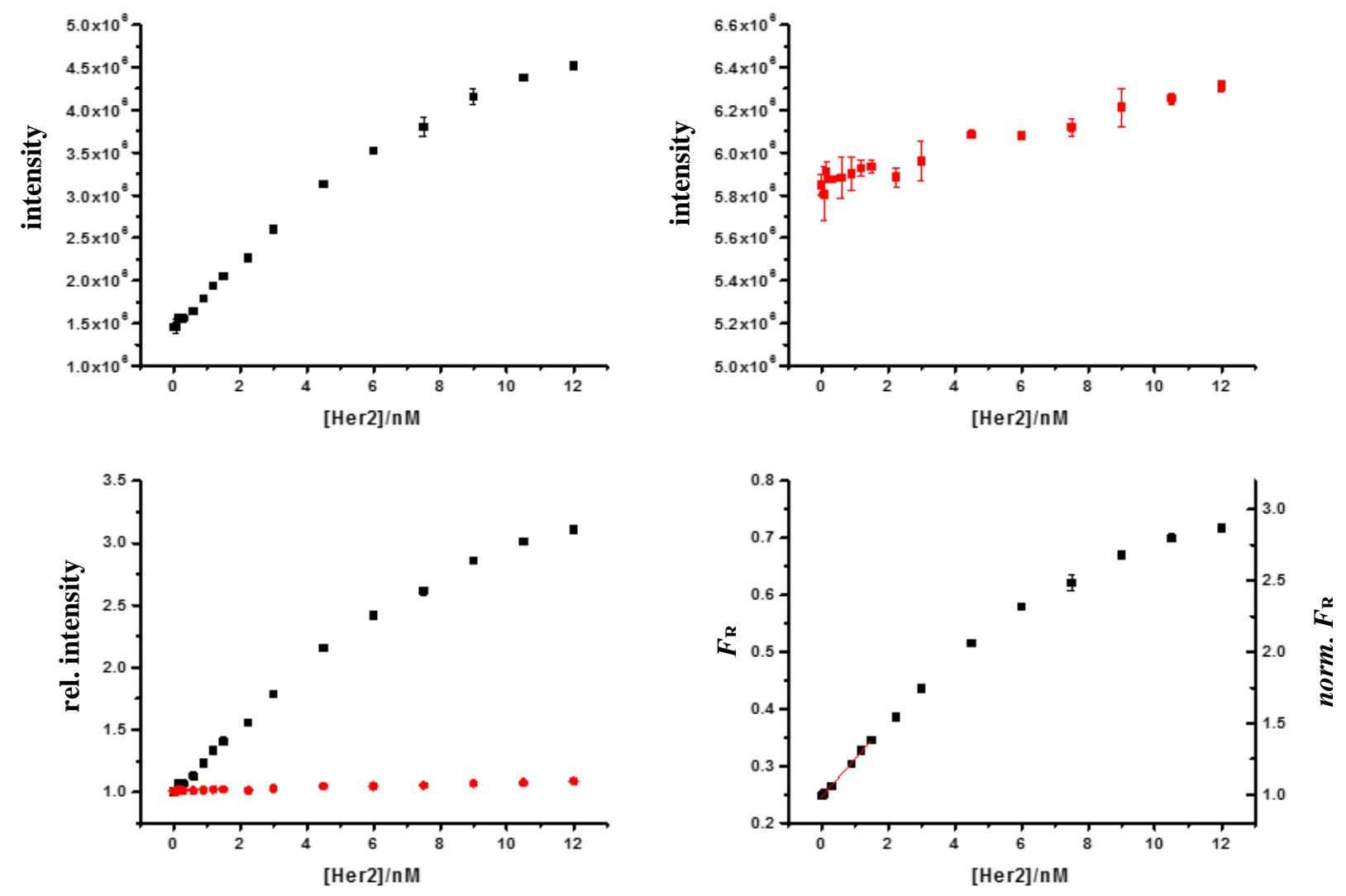

Figure S25: Tb-Tras-HER2-PertFab-QD650 immunoassay. Top left: Time-gated $(0.1-0.9 \mathrm{~ms}) \mathrm{PL}$ intensity in the QD channel over HER2 concentration. Top right: Time-gated $(0.1-0.9 \mathrm{~ms}) \mathrm{PL}$ intensity in the Tb channel over HER2 concentration. Bottom left: Both time-gated intensities normalized to unity at zero HER2. Bottom right: Linear part of the calibration curve that was used for calculating the LOD. 

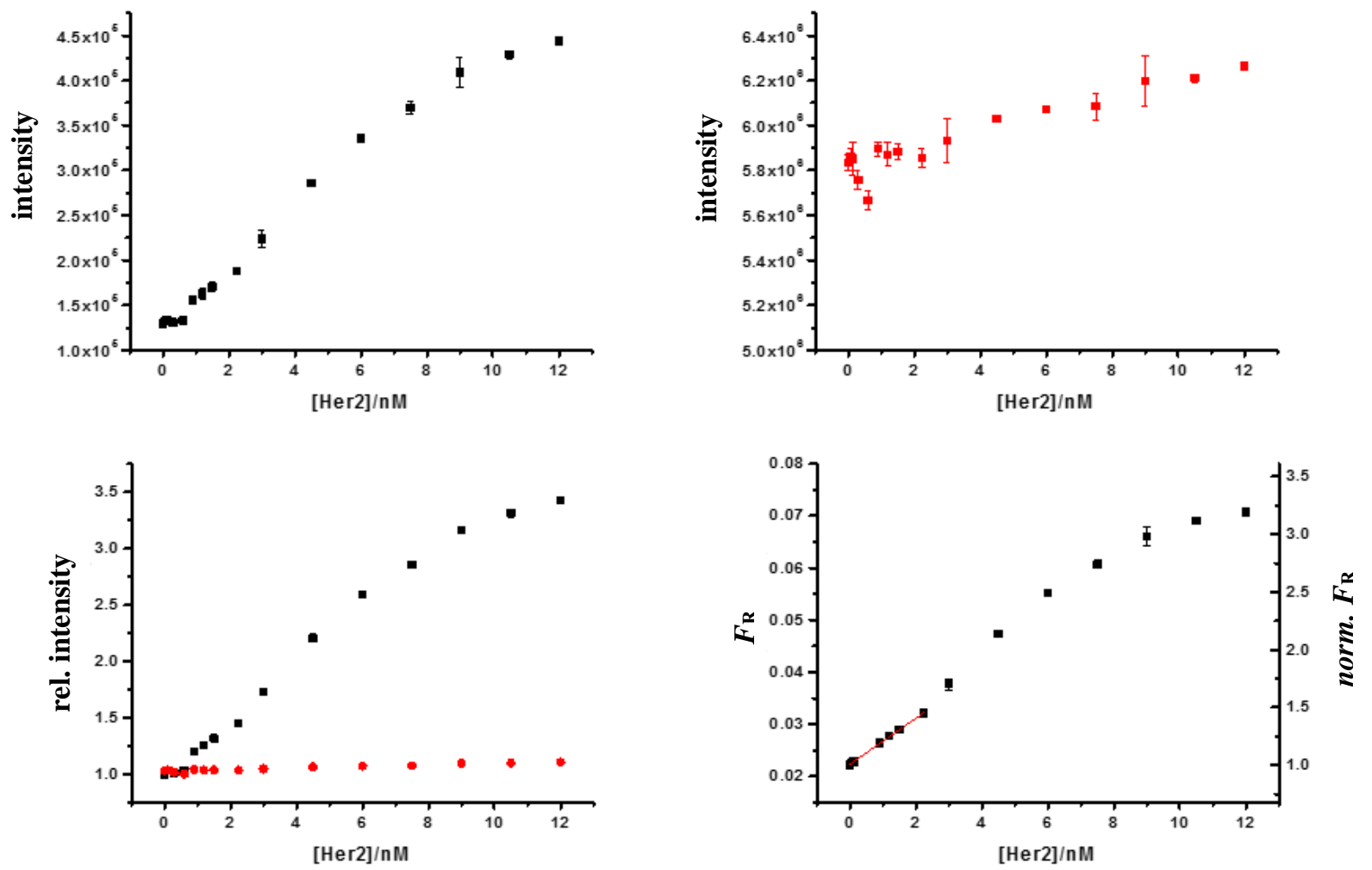

Figure S26: Tb-Tras-HER2-PertFab-QD605 immunoassay. Top left: Time-gated (0.1 - $0.9 \mathrm{~ms}) \mathrm{PL}$ intensity in the QD channel over HER2 concentration. Top right: Time-gated $(0.1-0.9 \mathrm{~ms}) \mathrm{PL}$ intensity in the Tb channel over HER2 concentration. Bottom left: Both time-gated intensities normalized to unity at zero HER2. Bottom right: Linear part of the calibration curve that was used for calculating the LOD.

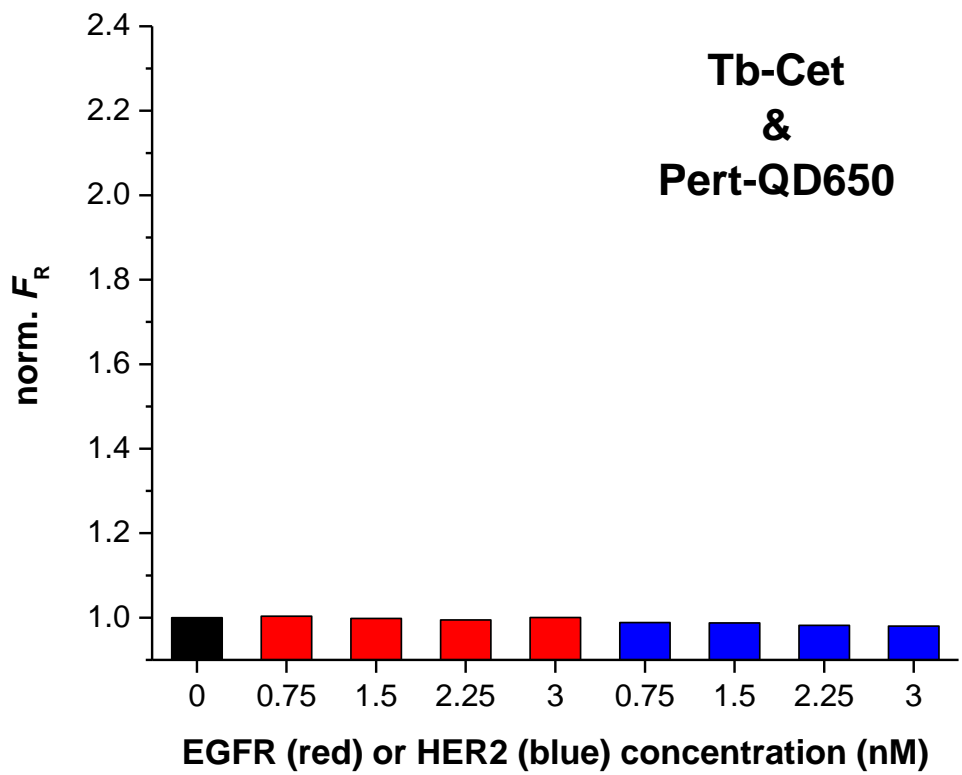

Figure S27: Tb-Cet / Pert-QD650 immunoassays show no EGFR or HER2 dependent FRET signals (Cet is specific for EGFR and Pert for HER2). At all measured concentration the $F_{\mathrm{R}}$ signals are similar to the background signal (no EGFR or HER2 in the sample). 